\title{
Balloon tamponade in variceal bleeding: use and misuse
}

\section{P Vlavianos, A E S Gimson, D Westaby, Roger Williams}

Liver Unit, King's College Hospital and School of Medicine and Dentistry, London SE5 9RS

$\mathrm{P}$ Vlavianos, $\mathrm{MD}$, research fellow

A E S Gimson, MRCP, senior registrar

D Westaby, MRCP, consultant physician and endoscopist Roger Williams, FRCP, director and consultant physician

Correspondence to: Dr Westaby.

BrMed J 1989;298:1158
The efficacy of and complication rates associated with balloon tamponade have been reported from centres experienced in using the procedure.$^{1.3}$ This prospective survey was carried out to assess the value and complication rates of balloon tamponade when used in hospitals with limited experience of managing acute variceal bleeding.

\section{Patients, methods, and results}

Over two years 30 patients were referred to this unit for the management of variceal bleeding with a balloon in position. Technical factors that we considered important for controlling bleeding were for the gastric balloon to be positioned close to the gastro-oesophageal junction and for it to be adequately inflated (in excess of $100 \mathrm{ml}$ ). Factors that we believed increased the risk of complications were: inflating the gastric balloon within the oesophagus (with the risk of oesophageal rupture); inadequately filling the gastric balloon $(<100$ $\mathrm{ml}$ allowed the tube to move upwards into the oesophagus, increasing the risk of occlusion of the larynx and pulmonary aspiration), inflating an oesophageal balloon at the level of the larynx; and using excessive mechanical traction $(>1 \mathrm{~kg})$ of the tube or applying inflation for prolonged periods $(>36$ hours).

In only 13 of the 30 patients was balloon tamponade effectively used to control bleeding. Failure to position the balloon correctly ( 13 cases) was the most common fault. In 15 cases the tube was used in such a way that there was a high risk of complications. The most common problem was underinflation of the gastric balloon (eight cases). In five cases excessive or prolonged traction was applied to the tube and resulted in severe ulceration of the gastro-oesophageal junction. In one case the gastric balloon was inflated in the oesophagus, but without serious consequences. Balloon tamponade was considered to be optimum - that is, both effective and safe-in only 10 of the patients.

\section{Comment}

We conclude that in most of these cases the balloon could not have stopped the bleeding and, furthermore, was likely to lead to complications. The most common error limiting the efficacy of tamponade was failure to position the gastric balloon correctly at the gastrooesophageal junction. This procedure needs the firm retraction of an adequately inflated gastric balloon back to the cardia (we use $160-200 \mathrm{ml}$ of a watercontrast mixture). The tube is then taped to the side of the face to provide skin traction and the position is checked radiologically. It is seldom necessary to inflate the oesophageal balloon if the gastric balloon is correctly positioned. Prolonged application of tamponade may cause severe mucosal ulceration, which can subsequently cause bleeding. This complication may be minimised by restricting the use of tamponade to less than 12 hours. Subsequent treatment should aim to prevent recurrent bleeding when the tube is withdrawn.

Guidelines for use cannot compensate for lack of experience, and our survey shows that without experience in its use balloon tamponade is of limited value. A pharmacological regimen ${ }^{4}$ in conjunction with careful resuscitation offers more chance of controlling bleeding and has a lower risk of complications than inexperienced use of balloon tamponade.

Balloon tamponade is always a thoroughly unpleasant experience for the patient; this survey has highlighted major limitations in its use, and there is a high risk of complications. ${ }^{5}$ These facts should lead hospitals to review their policy for managing variceal bleeding.

1 Rowntree LG, Zimmerman EF, Todd MH, Ajac J. Intra-oesophageal venous tamponade. Its use in a case of variceal hemorrhage from the esophagus. IAMA 1947;135:630-1.

2 Bauer JJ, Kreel I, Kork AE. The use of the Sengstaken-Blakemore tube for immediate control of bleeding oesophageal varices. Ann Surg 1974;179:273-7.

3 Sarin SK, Nundy S. Balloon tamponade in the management of bleeding oesophageal varices. Ann $R$ Coll Surg Engl 1984;66:30-2.

4 Burroughs AK. The management of bleeding due to portal hypertension. Part I. The management of acute bleeding episodes. Qf Med 1988;67:447-8.

5 Chojkier M, Conn HO. Esophageal tamponade in the treatment of bleeding varices. A decadal progress report. Dig Dis Sci 1980;25:267-72.

(Accepted 30 December 1988)
Department of Medicine (Elderly), St James's University Hospital, Leeds LS9 7TF

N D Penn, MRCP, tutor in medicine (elderly)

P W Belfield, MRCP, senior registrar

B H Mascie-Taylor, MRCP, consultant physician G P Mulley, FRCP, consultant physician

Correspondence to: $\mathrm{Dr}$ Penn.

BrMed $\mathcal{J}$ 1989;298:1158-9

\section{Old and unwashed: bathing problems in the over 70s}

\section{N D Penn, P W Belfield, B H Mascie-Taylor, G P Mulley}

Although bathing is an important aspect of self care, little information exists on the problems it causes in elderly people. We assessed the difficulties of bathing and identified what might be done to improve the provision of bathing for old people living at home.

\section{Patients, methods, and results}

On each of 200 consecutive domiciliary visits carried out in Leeds Eastern district by this department we recorded age, sex, ability to bathe, whether help was required, whether the patient lived with someone and if so what help was provided, and frequency of bathing.

Altogether 175 questionnaires, on 116 women and
59 men with a mean age of 83 (range 70-94), were suitable for analysis. Eighty one patients lived alone, 64 with their spouses, 11 with their daughters, and 19 with another carer, such as a niece or sister.

All homes had baths; nine also had showers, only three of which were used. Ninety five people were able to have a bath, 46 requiring help. As an alternative to bathing 59 people had a standing strip wash, 42 requiring help. Twenty one people were unable to have a bath or a strip wash. The table shows who provided the help. Spouses provided the same proportion of help

Provision of help with washing and bathing for elderly people

\begin{tabular}{lccc}
\hline & $\begin{array}{c}\text { People who } \\
\text { had baths } \\
(\mathrm{n}=46)\end{array}$ & $\begin{array}{c}\text { People who } \\
\text { had standing } \\
\text { strip washes } \\
(\mathrm{n}=42)\end{array}$ & $\begin{array}{c}\text { Total (\%) } \\
(\mathbf{n}=88)\end{array}$ \\
Who provided help & 13 & 11 & $24(14)$ \\
\hline Family and friends: & 12 & 9 & $21(12)$ \\
$\quad$ Spouse & 11 & 5 & $16(9)$ \\
Daughter & 7 & 17 & $24(14)$ \\
$\begin{array}{l}\text { Other } \\
\text { Health and social services: }\end{array}$ & & & $2(1)$ \\
$\quad$ Nurse & 2 & & $1(<1)$ \\
$\quad$ Care assistant & 1 & & \\
$\quad$ Home help & & & \\
\hline
\end{tabular}


as community nurses, and only slightly less help was provided by daughters. Overall, female relatives provided $61 \%(54 / 88)$ of all help. Two men helped their fathers to bathe, but no male relatives helped with strip washes. Only two patients had baths at day centres.

Seventy four people could bathe as often as they liked. Fifteen people (predominantly women with stroke or dementia) had not had a bath for over a year (maximum 18 years). Over half of the patients had been provided with one or more bath aids (seat, mat, rail, or board). Of the 220 aids, 66 were not in use and 13 had never been used.

\section{Comment}

Bathing presents the greatest number of problems to old people at home. ${ }^{1}$ In our study of 175 selected ill patients over the age of 70 only 95 were able to bathe, a proportion similar to that seen in previous studies. ${ }^{12}$ Forty nine were independent in bathing, the rest requiring personal help. Although nursing services provided this help in only 24 cases, bathing is the activity most frequently carried out by nurses and auxiliaries who visit people over the age of 75 at home. ${ }^{1}$ In some districts bathing is increasingly carried out by the social services homecare team. The frequency of bathing, however, is usually only once a fortnight, and all those people receiving such help in our survey thought that this was not enough. By contrast, the people who received help from their family or friends were bathed at least once a week. Only two people had baths at day centres. Although less cost effective than providing help with bathing at home, bathing at day centres allowed old people to enjoy a proper bath with help from care assistants.

Our finding that over half of the elderly people surveyed were not receiving baths as often as they would have liked merits fuller consideration. If personal help is needed then family or friends are usually willing to provide it. Some people, however, may prefer to seek professional help from either the community nursing team or social services.

We believe that personal hygiene is central to good health and wellbeing and recommend that any health professional assessing a disabled or elderly person should routinely ask about bathing. Sufficient services should be available to enable people to be bathed in their own home at least once a week.

We thank Dr W E Bagnall, Dr T R Srinivasan, and Dr J T Eccles for their help in collecting data.

1 Clark M, Clark S, Odell A, Jagger C. The elderly at home: health and social status. Health Trends 1984;16:3-7.

2 George J, Binns VE, Clayden AD, Mulley GP. Aids and adaptations for the elderly at home: underprovided, underused, and undermaintained. $\mathrm{Br} M \mathrm{Med}^{\mathcal{F}}$ 1988;296:1365-6

(Accepted 23 fanuary 1989)

\section{Forearm fractures in schoolchildren}

D Stanley, M J Bell

\section{Department of \\ Orthopaedics, Children's \\ Hospital, Sheffield \\ S10 2TH \\ D Stanley, FRCS, senior \\ registrar \\ M J Bell, FRCs, consultant}

Correspondence to:

$\mathrm{Mr}$ Stanley.

$\operatorname{BrMed} \mathcal{f}$ 1989;298:1159-60
In the United Kingdom, as elsewhere in the Western world, accidental injury is the most common cause of morbidity among schoolchildren' and accounts for up to one child in five presenting each year to accident and emergency departments. ${ }^{2}$ Fractures and sprains account for over a quarter of all childhood accidents ${ }^{2}$ and are a major cause of orthopaedic referral. Within this group fractures of the forearm figure prominently. ${ }^{3}$ Although accidental injury implies "an unpredictable and therefore unpreventable event," the magnitude of the problem led us to reconsider this definition.

\section{Patients, methods, and results}

We carried out a prospective study of 200 schoolchildren aged 5 to 15 who had fractured their forearm. The series was consecutive, with all children being seen in the fracture clinic at this hospital from January to June 1988. We recorded the age and sex of the child, the cause of the accident, whether the fracture required manipulation, and where the accident had happened.

Of the 200 children, 123 were boys and 77 girls. The table shows the causes of fracture. For both sexes the most common cause was running and tripping, with $40 \%$ of all fractures occurring at the weekend or during school holidays.

The incidence of fracture was higher in children not supervised by an adult $(115(58 \%))$, and fractures in this group also more often required manipulation under anaesthetic (48/70 (69\%)). Of all the manipulations performed, $60(85 \%)$ were carried out after $5 \mathrm{pm}$, which influences the hospital service that must be provided. Altogether $26 \%$ of fractures occurred at school, and, though supervision seemed adequate during organised physical education and sporting activities, there was little supervision during "break" periods, when most fractures occurred.

\section{Comment}

Children cannot be completely protected from accidents as such an environment would impede the development of their independence. ${ }^{4}$ Protecting children from things that are unnecessary for development, however, is worthy of attention.

We found that simple falls were the most common causes of fractures in both boys and girls, and reducing these would seem impossible to achieve. Thus simple falls most closely fit the definition of accidental injury. In all other cases a clear precipitant of fracture was found, particularly in girls who fell over while roller skating. Although the degree of experience of roller skating varied, none of them had received any formal instruction and all of them had sustained their injuries while playing locally on ground surfaces that were not ideal for this type of sport. The difference in rates of injury between girls on roller skates $(16 / 77,21 \%)$ and boys on skateboards $(8 / 123,7 \%)$ probably reflects the

Causes of fracture among 200 children attending fracture clinic over six months

\begin{tabular}{lcc}
\hline Cause of fracture & Boys $(\mathbf{n}=123)$ & Girls $(\mathbf{n}=\mathbf{7 7})$ \\
\hline Running and tripping & 22 & 22 \\
Falling from height & 16 & 1 \\
Playing football & 16 & 3 \\
Roller skating & 12 & 16 \\
Bicycling & 11 & 3 \\
Swinging on rope & 9 & \\
Playground accidents & 8 & 4 \\
Skateboarding & 8 & 3 \\
Fighting & 5 & 3 \\
Winter sports & 4 & 4 \\
Falling down stairs & 3 & 1 \\
Falling from chair & 3 & 6 \\
Physical education & 1 & \\
Road traffic accident & 5 & 11 \\
Minor sports & &
\end{tabular}

^Included basketball, judo, and karate for boys; and horse riding, rounders, netball, badminton, and gymnastics for girls. 